\title{
Aesthetic Rehabilitation with Nano- Ceramic Composite Material - Case Reports
}

\author{
Noor Hayati Azami ${ }^{1 *}$, Nora Sakina Mohd Noor ${ }^{1}$, Wei Pin Ngan ${ }^{1}$, Jovian Tze Yu Then ${ }^{1}$, Yeong \\ Chee Teoh ${ }^{1}$
}

KEYWORDS

nano-ceramic composite, direct restoration, Ceram. $\mathrm{X}$ one

\begin{abstract}
Aesthetic or cosmetic dentistry is one of the main areas of dental practice. Increasing demands of patients for aesthetics has resulted in the development of several techniques for restoring the anterior teeth. Composite resin restorations have become an integral part of contemporary restorative dentistry and can be called "star of minimal invasion" due to its conservative concepts. The direct composite veneering allows restoring the tooth in a natural way and preservation of sound tooth structure when compared to indirect restorations. Before placement of direct composite veneers, a proper diagnosis, available treatment options, cost of treatment, patient's expectations and duration of treatment should be taken into consideration. This article presents three case reports of aesthetic rehabilitation of anterior teeth using direct composite with satisfactory outcome that mimic the natural dentition.
\end{abstract}

\section{Introduction}

The restoration and re-establishment of a patient's dental aesthetics is among the important requirements of dentistry today [1,2]. Restorative dental practice has been expanded and revolutionized by recent developments in the field of adhesive dentistry [3-5]. Porcelain veneering is considered the gold standard as an aesthetic restorative option when used for suitable clinical cases [6-8]. The fabrication of porcelain veneers requires the removal of tooth structure, healthy or otherwise, to accommodate the porcelain.

This approach may be rather non-conservative and has led to the development of other techniques which aim to preserve tooth structure $[9,10]$. One such technique is the direct composite veneering. Better finishing and polishing can be carried out

\footnotetext{
${ }^{1}$ Department of Restorative Dentistry, Faculty of Dentistry, University of Malaya, 50603 Kuala Lumpur

*Correspondence: nhayatiazami@um.edu.my
}

with these composite resin materials and the results can be matched closely with porcelain [11,12]. Direct veneering with composite involves application of resin directly to the tooth structure and sculpted to correct aesthetic defects of color, anatomy and morphology [13]. Direct composite veneering has many advantages such as being a chair-side technique, not requiring multiple appointments, minimal application time required, minimally invasive, preserves natural tooth structure and less expensive due to no laboratory costs involved. Although direct composite veneering has quite a few advantages, yet it is prone to failure because it is highly technique sensitive and dependent on the skill of the clinician [14]. Also, failure is attributed to lack of durability when used in thick sections, changes in color and excessive wear [15]. Case selection is extremely important for the success of this direct restorative technique and ideal clinical indications include teeth discolorations, anatomical deformities, diastema, misaligned or worn dentition $[8,16]$. Teeth position and alignment determine the complexity of the case 
and a good and correct diagnosis and treatment plan is of paramount importance.

The clinician must possess a deep understanding of the concepts of chroma, value, hue, translucency and opacity in order to obtain an aesthetically pleasing and acceptable post veneering result [17]. The relationship of these concepts of the natural tooth structure with the physical and optical properties of composite resin materials is very crucial. The manipulation of composite resin and its placement and sculpting to resemble closely the natural form of teeth is an art and requires skills that can only be honed by extensive practice and patience. These three-clinical case reports present step-by-step sequence for restoring the aesthetics of anterior maxillary teeth with direct composite veneer technique.

\section{Case Presentation}

\section{Case 1}

A 25-year old female presented with concerns about spacing in her front teeth and yellowish canines which are causing her to have low confidence and low self-esteem. Teeth 12 and 22 are peg shaped laterals and were extracted for orthodontic purpose (Figure 1). The patient had opted for direct composite veneer restoration among the treatment options offered. Treatment plan: Multiple diastemas closure and to re-shape teeth 13 and 23 using direct composite veneer restoration using Ceram. $X^{\infty}$ one ${ }^{T m}$ (Dentsply, Konstanz, Germany) to mimic the missing lateral incisors.

The treatment procedures are as follows: -

1. Pre-operative smiling view was taken showing discoloured canines. (Figure 2A).

2. Diagnostic wax up was made to reproduce the desired morphology. A putty index was fabricated and used as a template (Figure 2B).

3. The shade $A 1$ was selected by placing composite button in the incisal third of 21 and shade $A 2$ in the middle third of 13 (Figure 2C).

4. Maxillary anterior region was isolated with rubber dam. Caries at mesial surface of 23 was removed. Teeth were cleaned with pumice (Figure 2D).

5. Adjacent teeth were isolated with PTFE tape. DeTrey Conditioner 36 was applied for 15s, followed by rinsing with water spray. Prime\&Bond ${ }^{\circ}$ one SELECT was applied for 20s, blown with air syringe for $5 \mathrm{~s}$, and light cured for 10 s (Figure $2 \mathrm{E}$ ).

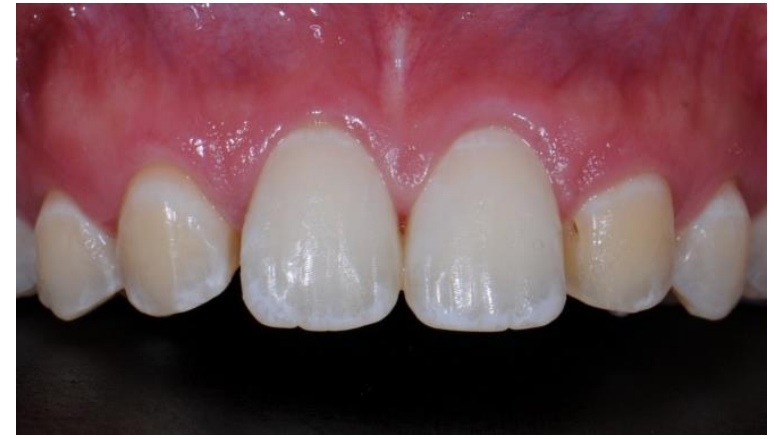

Figure 1. Retracted preoperative view showed diastemas between 13-11 and 21-23. Mesial caries and labial composite restoration on 23 was observed.
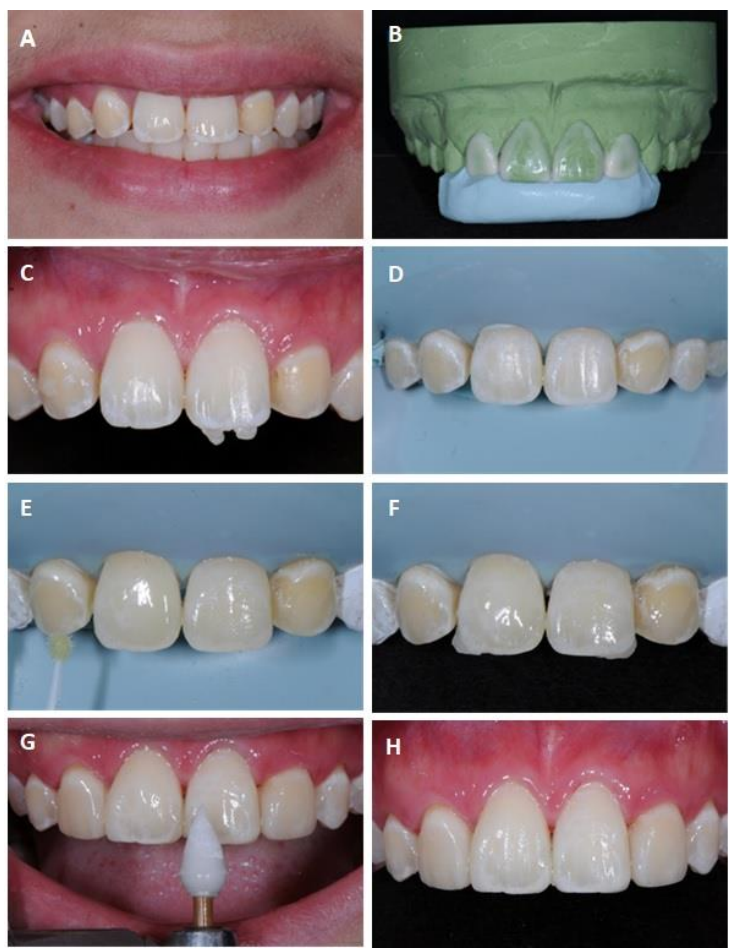

Figure 2. The treatment procedures are as following; (A) Smiling pre-operative view showed mismatch color of tooth 23 and multiple diastemas.

(B) Diagnostic wax-up (C) Shade selection using composite button prior to rubber dam placement. (D) Multiple teeth isolation with rubber dam. (E) Teeth surfaces after etching and bonding. (F) Palatal shelves build up using the putty index. (G) Finishing and polishing. $(\mathrm{H})$ Final result showing excellent soft tissue contours and color integration of the Ceram. $\mathrm{X}$ composite restoration.

6. Palatal composite shelves were formed using silicone key. Labial surfaces were sculpted using Ceram. $X^{\oplus}$ one (A1). Same procedure was carried out on the remaining teeth (Figure $2 \mathrm{~F}$ ). 
7. Excess material in the cervical and proximal area were removed and contoured. Finishing and polishing were accomplished using Enhance PoGo Polishing System combined with Prisma Gloss $^{\mathrm{Tm}}$ (Figure $2 \mathrm{G}$ ).

8. Final result of direct composite restoration showed excellent colour match and natural appearance.

9. Postoperative view showed the complete diastemas closure. Teeth 13 and 23 were reshaped to mimic the lateral incisors with good colour match using direct composite veneer restorations (Figure 2H).

\section{Case 2}

A 21-year old female came to the Department of Restorative Dentistry, complaining of large gap between her upper front teeth (Figure 3). She refused to have orthodontic and prosthodontic treatments. The clinical examination showed multiple diastemas between teeth 13-12, 11-21, 22-23. She has a Class III incisor relationship (edge to edge). Treatment plan: Diastema closure with direct composite restoration using Ceram. $X^{\circledR}$ One (Dentsply, Konstanz, Germany).

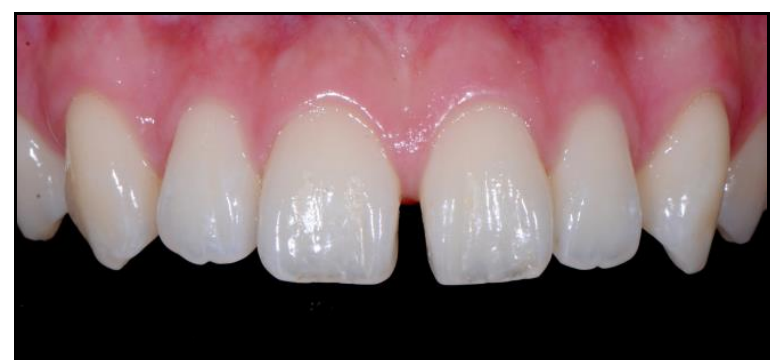

Figure 3. Preoperative intraoral view showed multiple diastemas between teeth 13-12, 11-21 and 22-23.

The treatment procedures are as follows (see Figure 4A-H): -

1. Study model and diagnostic wax up was prepared, and a silicone putty index was used to duplicate the mock-up and later used as palatal matrix (Figure 4A)

2. The shade was selected by placing composite buttons $(1.5 \mathrm{~mm})$ in the middle to select dentine shade and incisal third for enamel shade. Shade A2 and $A 1$ was selected for dentine and enamel respectively (Figure $4 B$ ).

3. Multiple teeth isolation was done from tooth 13 to 23 with rubber dam. Teeth surfaces were cleaned with pumice and water using rubber cup. Tooth preparation was not required (Figure 4C).

4. Isolation of adjacent teeth with PTFE tape. Tooth 12 to 22 were etched with DeTrey ${ }^{\circledR}$ Conditioner for $15 \mathrm{~s}$ then rinsed with water spray and blot dried. Prime\&bond ${ }^{\circledR}$ one SELECT was applied with rubbing motion, blowed with air syringe and light cured for 20s (Figure 4D).

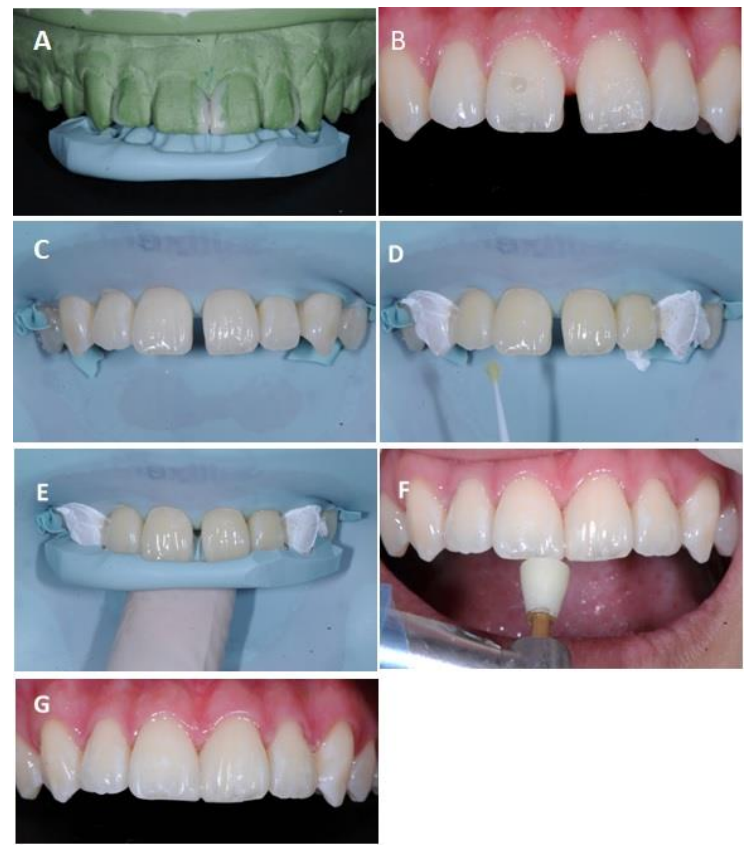

Figure 4. The treatment procedures for case 2 are as follows; A) Diagnostic wax-up and putty index in-situ; B) Shade selection using composite button technique; C) Multiple teeth isolation; D) Etching \& bonding; E) Silicone putty index adaptation; F) Finishing and polishing, G) Final result.

5. A silicone key was used to identify the correct palatal extension of the restoration. Composite with selected shades were adapted to fill up the missing space (layering technique) (Figure 4E).

6. Rubber dam was removed. Occlusion checked. Finishing and polishing were completed with Enhance ${ }^{\circledR}$ Finishing and $\mathrm{PoGo}^{\circledR}$ Polishing System combined with Prisma ${ }^{\circledR}$ Gloss (Figure 4F).

7. Final result showed complete diastema closure. The composite restoration was contoured to give natural appearance and harmonious integration of Ceram.X One ${ }^{\circledR}$ (Figure 4G).

\section{Case 3}

A 23-year old male came to the Department of Restorative Dentistry, with the complaint of 
fractured upper front teeth, as a result of a fall when he was 13-years old (Figure 5). He currently has an aesthetic concern over his fractured teeth leading to low self-esteem. He would like to improve the appearance of his upper anterior teeth which would then restore his confidence. Clinical examination showed both central incisors were fractured involving the incisal edges and incisal angles, the pulp was not involved on both teeth. Tooth 21 is mal-positioned mesiolabialy. Treatment plan: Restore fractured teeth and correct the alignment with direct composite restoration using Ceram. $X^{\circledR}$ one.

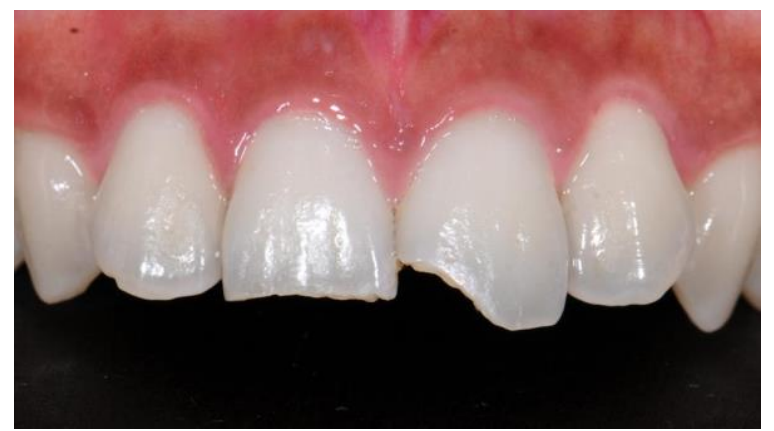

Figure 5. Retracted pre-operative intraoral view showed non-complicated crown fracture on tooth 11 and 21 and slightly rotated tooth 21 .

The treatment procedures were as follows: -

1. Pre-operative maxillary central incisors occlusal view showed the misaligned central incisors before correction with the composite build-up. The shape and contour of the restoration were discussed to achieve the anterior teeth aesthetic (Figure 6A).

2. The diagnostic mock-up was made to establish the shape and alignment of the restorations using the composite resin. A putty index was used to duplicate the mock-up and used as a silicone key (Figure 6B).

3. Prior to rubber dam isolation, shade selection was done. Shade A2 and A1 was selected for body and incisal shades respectively. Multiple teeth isolation was done from tooth 13 to 23 with rubber dam. Teeth surfaces were cleaned with pumice and water using rubber cup. Tooth 11 and 21 were prepared by bevelling with diamond bur for retention and aesthetic blending at the toothrestoration interface (Figure $6 \mathrm{C}$ ).

4. After etching and bonding, the silicone key was used to construct palatal shells and proximal extensions. Composite with selected shades (A1 for incisal and A2 for body) were applied using layering technique (Figure 6D).
5. After contouring and removal of the excess material, finishing and polishing were completed with Enhance ${ }^{\circledR}$ Finishing and $\mathrm{PoGo}^{\oplus}$ Polishing System combined with Prisma ${ }^{\circledR}$ Gloss (Figure 6E).

6. Post-operative maxillary central incisors occlusal view showed the corrected alignment and aesthetic morphology (Figure 6F).

7. Post-operative retracted intraoral view showed the natural appearance of the direct composite restoration with Ceram. $X^{\oplus}$ one and the corrected alignment of the tooth 11 and 21 (Figure 6G).
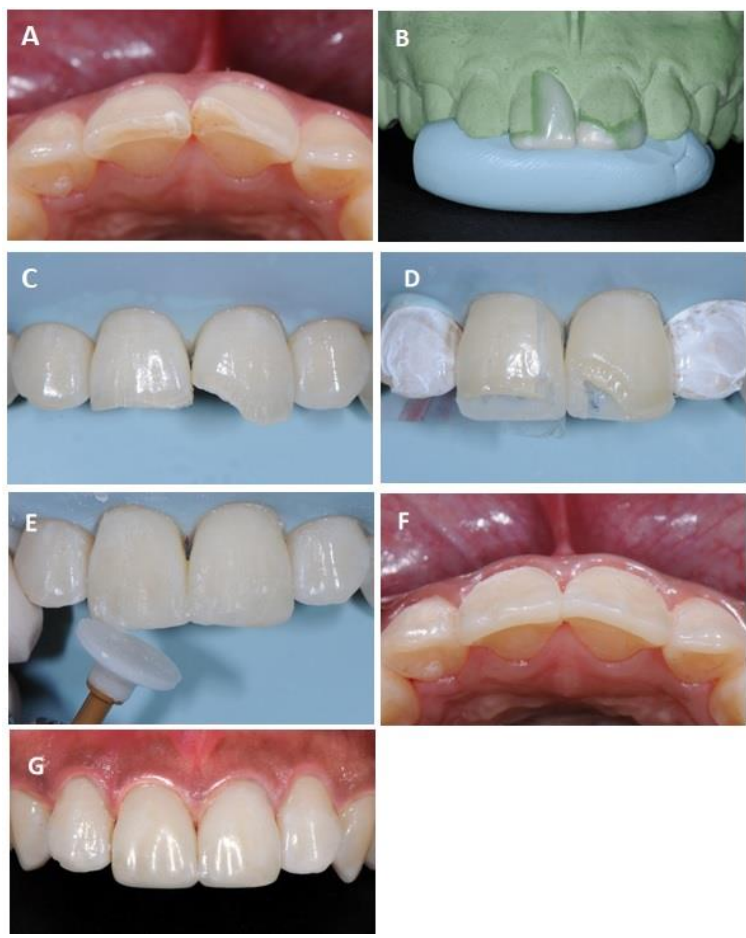

Figure 6. The treatment procedures for case 3 are as follows; A) Occlusal view showing the mildly rotated 21; B) Mock up and silicone putty index; C). Teeth preparation after bevel.; D) Palatal wall build-up and layering technique.; E) Finishing and polishing; F) Occlusal view; G) Final result

\section{DISCUSSION}

Shade matching of final restorative materials can provide a challenge in many cases, but Ceram.X one provides a unique and innovative solution designed to make shade matching fast and easy with predictable outcomes. Ceram.X one uses just five CLOUD shades of intermediate translucency, cleverly designed to shade match all 16 VITA classic shades. The shade of Ceram.X one restorations is influenced by the color of the surrounding tooth structure; a phenomenon known as the 'chameleon effect' meaning that Ceram.X one's CLOUD shades are able to cover 
more than one VITA shade. There is also one additional shade especially designed for the restoration of whitened teeth.

Ceram.X one is based on the advanced granulated filler technology SphereTEC ${ }^{\text {TM }}$ which is patent applied. SphereTEC ${ }^{\text {тM }}$ stands for the process of manufacturing microscaled, well-defined spherical superstructures, comprising of submicron glass. The SphereTEC ${ }^{\text {тM }}$ fillers' morphology, particle size distribution and surface microstructure are key for the enhanced handling comfort and aesthetic outcome.

The unique sub-micron particles within Ceram.X one gives superior aesthetics and wear resistance, leading to fast polishing and extra gloss on the finished restoration. Ceram. $X$ one ${ }^{\circledR}$ provides a fast and easy polishing procedure with both, siliconebased polishers and abrasive discs, resulting in an outstanding gloss. During this procedure the sub- micron particles of the SphereTEC filler are removed layer by layer, thereby not impacting the gloss surface and leading to an even, postpolishing surface.

\section{CONCLUSION}

There are several approaches to close multiple diastemas including orthodontics, indirect restoration and direct composite restoration. Direct composite restorations were chosen because it enables a fast aesthetic rehabilitation in a single appointment and non-invasive teeth preparation. Ceram. $X^{\circledast}$ one showed remarkable highly aesthetic outcomes and most importantly it satisfied the patient's expectation.

\section{REFERENCES}

1. Korkut, B.; Yanıkoğlu, F.; Günday, M. Direct composite laminate veneers: Three case reports. Journal of dental research, dental clinics, dental prospects 2013; 7: 105.

2. Goldstein, R.E. Esthetics in dentistry (2 vols). PMPH-USA: 2014.

3. Haller, B. Recent developments in dentin bonding. American Journal of dentistry 2000; 13: 44-50.

4. Perdigao, J. New developments in dental adhesion. Dental clinics of North America 2007; 51: 333-357.

5. Summitt, J.B.; dos Santos, J. Fundamentals of operative dentistry: A contemporary approach. Quintessence Pub.2006.

6. Peumans, M.; Van Meerbeek, B.; Lambrechts, P.; Vanherle, G. Porcelain veneers: A review of the literature. Journal of dentistry 2000; 28: 163-177.

7. Calamia, J.R.; Calamia, C.S. Porcelain laminate veneers: Reasons for 25 years of success. Dental clinics of North America 2007; 51: 399-417.

8. Lim, C.C. Case selection for porcelain veneers. Quintessence international (Berlin, Germany: 1985) 1995; 26: 311-315.

9. Gurel, G. Science and art of porcelain laminate veneers. Quintessence Publishing (IL): 2003.

10. Heymann, H.O.; Swift Jr, E.J.; Ritter, A.V. Sturdevant's art \& science of operative dentistry. Elsevier Health Sciences: 2014.

11. Fahl, N. A polychromatic composite layering approach for solving a complex class iv/direct veneer/diastema combination: Part ii. Practical Procedures and Aesthetic Dentistry, 2007;19: 17.

12. Fahl Jr, N. Achieving ultimate anterior esthetics with a new microhybrid composite. Compend Contin Educ Dent Suppl, 2000; 26: 4-13.

13. Garber, D. Direct composite veneers versus etched porcelain laminate veneers. Dental clinics of North America, 1989; 33: 301-304.

14. Sadowsky, S.J. An overview of treatment considerations for esthetic restorations: A review of the literature. The Journal of prosthetic dentistry, 2006; 96: 433-442.

15. Hemmings, K.W.; Darbar, U.R.; Vaughan, S. Tooth wear treated with direct composite restorations at an increased vertical dimension: Results at 30 months. The Journal of prosthetic dentistry 2000, 83, 287-293.

16. Fahl, J.N. The direct/indirect composite resin veneers: A case report. Practical periodontics and aesthetic dentistry: PPAD 1996, 8: 627-638

17. Titus, H.; Chan, D. A comparison of gingival marginal gap formation in composite inlays using three different fabrication techniques. Texas Dental Journal, 1992; 109:13-16.

License Information: This work is licensed under a Creative Commons Attribution 